\title{
Delivery of the Porcupine Inhibitor WNT974 in Mice
}

\author{
Li-shu Zhang and Lawrence Lum
}

\begin{abstract}
We describe here a technique for delivering the porcupine inhibitor WNT974 (formerly LGK974) in mice. The protocol entails once-a-day oral delivery of WNT974 for up to 3 months at a concentration sufficient to achieve systemic Wnt pathway inhibition with limited toxicity as measured by weight change. This route of delivery enables extended durations of Wnt signaling inhibition in a mammalian model organism.
\end{abstract}

\section{Keywords}

Porcupine; Wnt974/LGK974; Mice; Drug delivery

\section{Introduction}

WNT974 (also known as LGK974) is a first-in-class picomolar inhibitor of the Wnt acyltransferase porcupine (Porcn) (Fig. 1) [1]. The compound is currently being evaluated as an anticancer agent against a broad range of diseases associated with deviant Wnt signaling. Although some cancer-associated mutations such as loss of the Wnt receptor ubiquitin ligase RNF43 are anticipated to promote Wnt ligand-mediated signaling in certain types of cancers [1-5], an incomplete inventory of Wnt pathway regulators suggests that genetic mutations yet to be unidentified may give rise to tumors that would be responsive to Porcn inhibitors. At the same time, regenerative medicine goals may see advances with the help of Wnt pathway modulators such as WNT974 by facilitating wound healing [6]. Thus, preclinical testing of compounds such as WNT974 in mouse models of disease is critical to a broader understanding of chemical utility.

A longstanding concern associated with chemically disabling Wnt signaling is the compromising of the intestinal epithelial lining that depends on Wnt ligands for renewal [7]. Although suppression of Wnt pathway activity with inhibitors targeting the Tankyrase enzymes which indirectly control $\beta$-catenin abundance by regulating the stability of its destruction complex is anticipated to have a poor therapeutic index, porcupine inhibitors appear to be relatively well tolerated [8, 9]. Thus, Porcn inhibitors such as WNT974 enable a variety of preclinical studies focused on Wnt signaling while limiting the potential influence of unwanted side effects on test outcomes. This protocol will provide an accessible approach for delivering WNT974 in animals by gavage and should be useful for a broad range of preclinical studies in mice that require short- or long-term suppression of Wnt-mediated signaling. 


\section{Materials}

\subsection{Drug Preparation}
1.
WNT974 (see Note 1). Store at $-20{ }^{\circ} \mathrm{C}$.
2. Dimethylacetamide (DMA).
3. Cremophor EL.
4.
Tween 80 .
5.
Dextrose solution: $5 \%$ Dextrose (w/v) in distilled water. Store at $4{ }^{\circ} \mathrm{C}$.
6.
Carboxymethylcellulose solution: $5 \%$ Carboxymethylcellulose (w/v) added to the dextrose solution. Store at $4{ }^{\circ} \mathrm{C}$.
7. $\quad 50 \mathrm{ml}$ Conical tubes.
8. $\quad 3.7 \mathrm{ml}$ Glass vials with screw thread.
9. Analytical balance, readability $0.01 \mathrm{mg}$.
10. Vortex mixer.

\subsection{Drug Delivery}

1. Mice, normal or disease models (see Note 2).

2. Feeding needles, plastic or metal.

3. $1 \mathrm{ml}$ Disposable syringes.

4. Soap solution: Add $1 \mathrm{ml}$ hand soap to $49 \mathrm{ml}$ distilled water, and mix well.

5. Ethanol solution: Add $35 \mathrm{ml} 100 \%$ ethanol to $15 \mathrm{ml}$ distilled water, and mix well.

\section{Methods}

An animal protocol must be approved by the IACUC prior to the start of WNT974 delivery in vivo. Animal research training and badge access to the animal facility are also required. For this protocol, we will use as an example 20 mice randomly divided into two cohorts henceforth referred to as control or WNT974 group. In addition, we will be dosing animals at $5 \mathrm{mg} / \mathrm{kg}$.

\subsection{Drug Preparation}

1.

Randomly group animals into two cohorts. Weigh each animal and calculate the average body weight (BW) for each group one day before

\footnotetext{
${ }^{1}$ When planning the experiment with the compound WNT974, it is advisable to purchase enough compound for the entire experiment to avoid the batch-to-batch differences. The activity of the compound should be tested in vitro before in vivo administration to ensure activity. Two routine methods can be used to evaluate WNT974 activity: detecting Wnt-induced transcriptional responses using culture cells expressing Wnt3a and harboring the Wnt-specific reporter known as Super Top Flash (STF reporter assay) [10] or monitoring levels of phospho-Dvl2 protein in a cell line such as HeLa cells that have cell-autonomous Wnt signaling [11].

${ }^{2}$ For gavaging 4- to 6-week-old mice, one should use smaller feeding needles (see Fig. 2) to avoid possible trauma to the esophagus. For gavaging mice younger than 4 weeks old, one may expect loss of some animals.
} 
dosing begins. For example, we will use an average body weight of $25 \mathrm{~g}$ for each group.

2.

Calculate the amount of WNT974 required for one dose using the average body weight: $5 \mathrm{mg} / \mathrm{kg} \times(25 \mathrm{~g} / 1000)=0.125 \mathrm{mg}$. The dosing volume for each mouse is $200 \mu$ l. Keep in mind that animal weight may change, thus necessitating modulation of daily total compound needs as the study progresses.

3. Remove WNT974 from a $-20{ }^{\circ} \mathrm{C}$ freezer and wait until the vial reaches room temperature. Weigh sufficient amount of compound for the total number of animals to be injected plus one extra dose in case of drug loss using the calculation from "2." Place compound into a glass vial (see Note 3). For the example used in this protocol, this would be $0.125 \mathrm{mg} \times 11=$ $1.375 \mathrm{mg}$.

4. Dropwise add DMA with gentle vortexing to achieve a final concentration of $5 \%$. In this case, since we dissolve $1.375 \mathrm{mg}$ of WNT974 into a total volume of $2.2 \mathrm{ml}$, this would be equal to $5 \% \times 2.2 \mathrm{ml}=121 \mu \mathrm{l}$. The solution should be clear.

5. Dropwise add Cremophor EL with gentle vortexing to a final concentration of $9 \%$ to the WNT974 in DMA. In this case, that would be $217.8 \mu \mathrm{l}$.

6.

Dropwise add Tween 80 with gentle vortexing to the WNT974/DMA/ Cremophor EL solution to achieve a final $1 \%$ concentration. In this case, this would be $24.2 \mu \mathrm{l}$.

7. Dropwise add carboxymethylcellulose solution with gentle vortexing to the WNT974/DMA/Cremophor EL/Tween solution to reach a final $85 \%$ concentration. In our example, this would be $2057 \mu$ l. The WNT974 solution should be used within $2 \mathrm{~h}$ of preparation.

8. Prepare the vehicle solution (without WNT974) for the control group similarly. The vehicle solution should be used within $2 \mathrm{~h}$ of preparation.

\subsection{Drug Delivery}

1. Don appropriate personal protective apparel as required by the animal facility prior to entering the facility (facemask, gown, booties, gloves, etc.).

2. Disinfect both gloved hands and the surface area one will work on.

\footnotetext{
${ }^{3}$ After calculating the amount of WNT974 for each group per dosing, we measure out the total WNT974 powder directly into a glass vial placed on an analytical balance. Of course, zero the balance with the empty glass vial first before adding compound. For convenience, prepare 12 aliquots each sufficient for a day's worth of dosing. The aliquots can be stored at $-20^{\circ} \mathrm{C}$. Reserve an extra amount of WNT974 for each dose in order to compensate for drug loss and changes in average animal weight. Typically, we include additional compound sufficient for two extra animals per aliquot.
} 
3. Draw $1 \mathrm{ml}$ of the WNT974 or vehicle solution into a $1 \mathrm{ml}$ syringe attached to a feeding needle (Fig. 2). Dislodge the air bubbles by gently tapping the side of the syringe with the feeding needle pointing upwards.

4.

Take one mouse out of the cage. Hold the mouse upright with one hand (Fig. 3). Insert the feeding needle gently into the mouth with the other hand. Allow the needle to slide into the esophagus and the opening of the stomach (see Note 4).

5. Push the plunger with the forefinger slowly but firmly to inject $200 \mu \mathrm{l}$ of the solution into the stomach.

6. Remove the needle and release the animal back into its cage.

7. Wash the needles after all of the mice have been gavaged. Flush the needles four to six times each in the following sequence: $2 \%$ soap solution, distilled water, and $70 \%$ ethanol. Store the needles in clean 50 $\mathrm{ml}$ conical tubes for future use.

8. Repeat this procedure for the total number of days desired. We have dosed mice daily (6 days a week) with $5 \mathrm{mg} / \mathrm{kg}$ WNT974 for up to 3 months without noticeable toxicity as measured by weight loss.

9.

Weigh mice three times a week. Do not forget to adjust the amount of WNT974 required per dose according to weight change in animals.

\section{Acknowledgement}

This work was supported by the NIH (R01 CA168761, R01CA196851), CPRIT (RP130212), and the Welch Foundation (I-1665).

\section{References}

1. Liu J, Pan S, Hsieh MH, Ng N, Sun F, Wang T, Kasibhatla S, Schuller AG, Li AG, Cheng D, Li J, Tompkins C, Pferdekamper A, Steffy A, Cheng J, Kowal C, Phung V, Guo G, Wang Y, Graham MP, Flynn S, Brenner JC, Li C, Villarroel MC, Schultz PG, Wu X, McNamara P, Sellers WR, Petruzzelli L, Boral AL, Seidel HM, McLaughlin ME, Che J, Carey TE, Vanasse G, Harris JL. Targeting Wntdriven cancer through the inhibition of porcupine by LGK974. Proc Natl Acad Sci U S A. 2013; 110:20224-20229. [PubMed: 24277854]

2. Boulter L, Guest RV, Kendall TJ, Wilson DH, Wojtacha D, Robson AJ, Ridgway RA, Samuel K, Van Rooijen N, Barry ST, Wigmore SJ, Sansom OJ, Forbes SJ. WNT signaling drives cholangiocarcinoma growth and can be pharmacologically inhibited. J Clin Invest. 2015; 125:12691285. [PubMed: 25689248]

3. Jiang X, Hao HX, Growney JD, Woolfenden S, Bottiglio C, Ng N, Lu B, Hsieh MH, Bagdasarian L, Meyer R, Smith TR, Avello M, Charlat O, Xie Y, Porter JA, Pan S, Liu J, McLaughlin ME, Cong F. Inactivating mutations of RNF43 confer Wnt dependency in pancreatic ductal adenocarcinoma. Proc Natl Acad Sci U S A. 2013; 110:12649-12654. [PubMed: 23847203]

4. Koo BK, van Es JH, van den Born M, Clevers H. Porcupine inhibitor suppresses paracrine Wntdriven growth of Rnf43;Znrf3-mutant neoplasia. Proc Natl Acad Sci U S A. 2015; 112:7548-7550. [PubMed: 26023187]

\footnotetext{
${ }^{4}$ Take the feeding needle out of the mouth if one feels blockage. Release the mouse and hold it again to let the needle slide into the mouse. It is strongly advised to practice gavaging prior to initiating an experiment. Holding the mouse upright firmly but not too tightly is key to successful gavaging.
} 
5. Madan B, Ke Z, Harmston N, Ho SY, Frois AO, Alam J, Jeyaraj DA, Pendharkar V, Ghosh K, Virshup IH, Manoharan V, Ong EH, Sangthongpitag K, Hill J, Petretto E, Keller TH, Lee MA, Matter A, Virshup DM. Wnt addiction of genetically defined cancers reversed by PORCN inhibition. Oncogene. 2015; 35:2197-2207. [PubMed: 26257057]

6. Lum L, Chen C. Chemical disruption of Wnt-dependent cell fate decision-making mechanisms in cancer and regenerative medicine. Curr Med Chem. 2015; 22:4091-4103. [PubMed: 26310918]

7. Clevers H, Nusse R. Wnt/beta-catenin signaling and disease. Cell. 2012; 149:1192-1205. [PubMed: 22682243]

8. Proffitt KD, Madan B, Ke Z, Pendharkar V, Ding L, Lee MA, Hannoush RN, Virshup DM. Pharmacological inhibition of the Wnt acyltransferase PORCN prevents growth of WNT-driven mammary cancer. Cancer Res. 2012; 73:502-507. [PubMed: 23188502]

9. Zhong Y, Katavolos P, Nguyen T, Lau T, Boggs J, Sambrone A, Kan D, Merchant M, Harstad E, Diaz D, Costa M, Schutten M. Tankyrase inhibition causes reversible intestinal toxicity in mice with a therapeutic index < 1. Toxicol Pathol. 2015; 44:267-278. [PubMed: 26692561]

10. Chen B, Dodge ME, Tang W, Lu J, Ma Z, Fan CW, Wei S, Hao W, Kilgore J, Williams NS, Roth MG, Amatruda JF, Chen C, Lum L. Small molecule-mediated disruption of Wnt-dependent signaling in tissue regeneration and cancer. Nat Chem Biol. 2009; 5:100-107. [PubMed: 19125156]

11. Dodge ME, Moon J, Tuladhar R, Lu J, Jacob LS, Zhang LS, Shi H, Wang X, Moro E, Mongera A, Argenton F, Karner CM, Carroll TJ, Chen C, Amatruda JF, Lum L. Diverse chemical scaffolds support direct inhibition of the membrane-bound O-acyltransferase porcupine. J Biol Chem. 2012; 287:23246-23254. [PubMed: 22593577] 


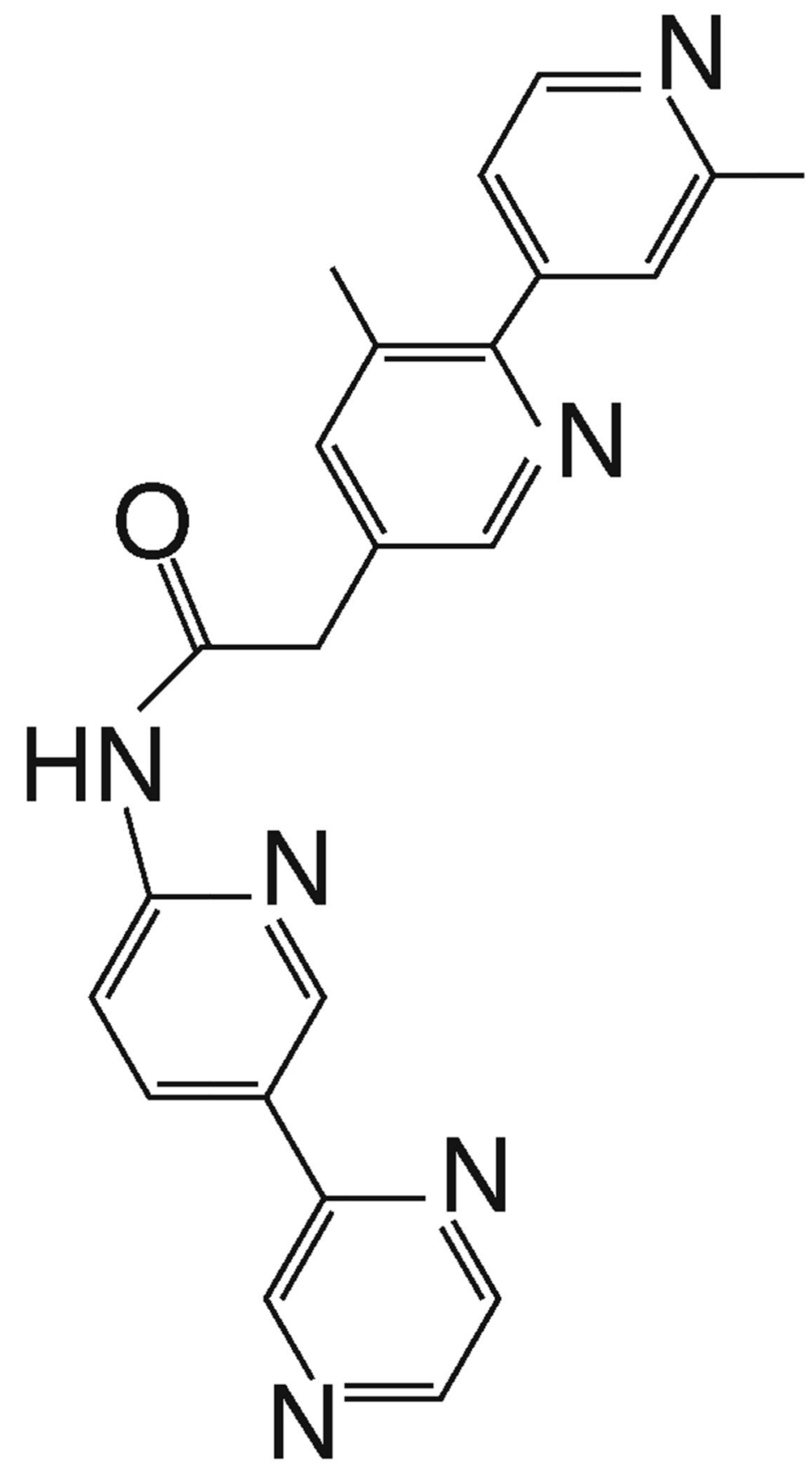

Fig. 1.

The chemical structure of WNT974 


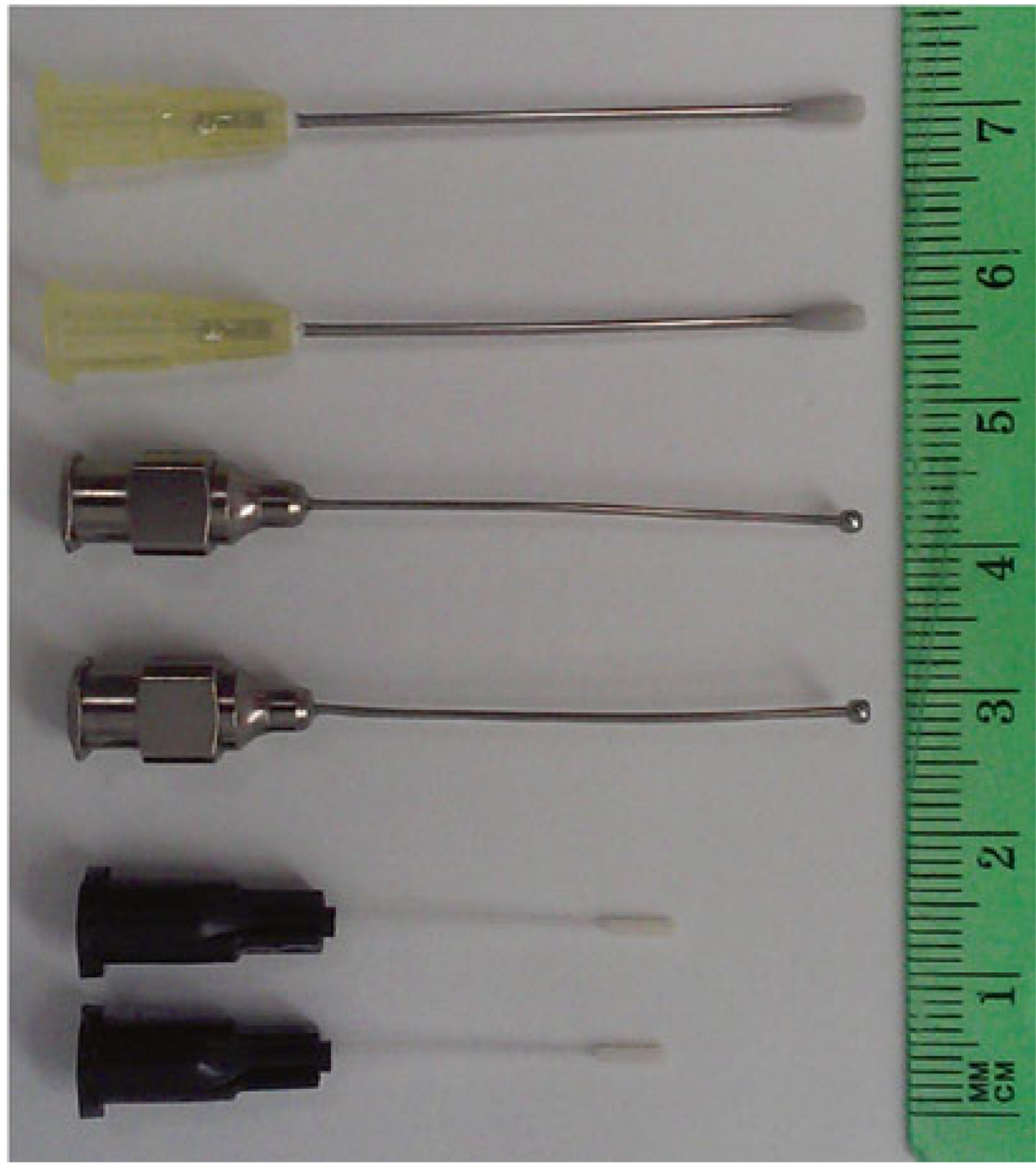

Fig. 2.

Feeding needles. Three different types of feeding needles are shown. The upper two needles are disposable and the middle two are reusable. Both are suitable for dosing mice older than 6 weeks old. The lower two needles are disposable and suitable for dosing mice younger than 6 weeks old 

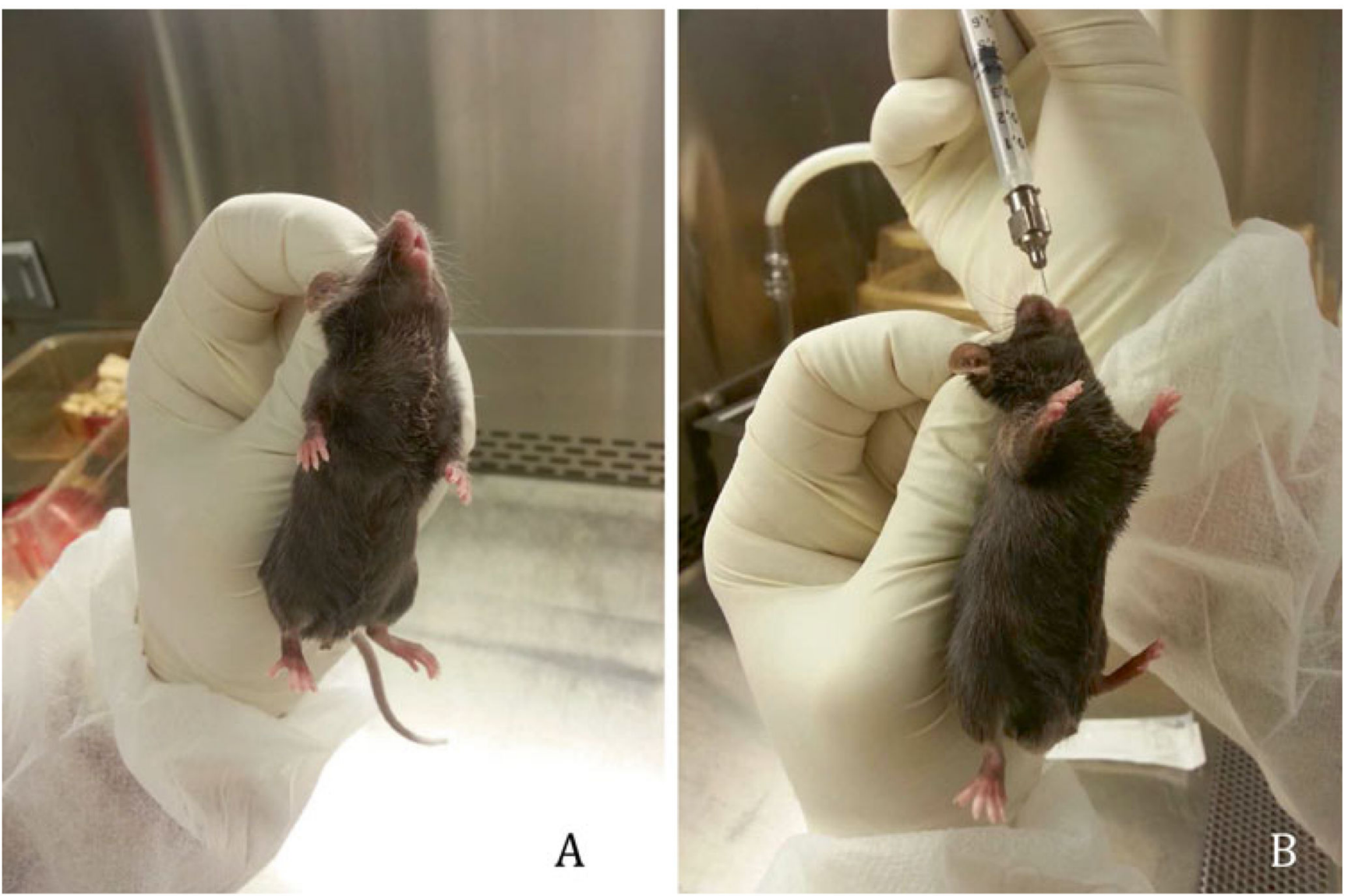

Fig. 3.

Oral gavaging. Holding mouse firmly (a) is the most important step for oral gavage. If the mouse's head or body moves freely, one will have to release and hold the mouse again. Next, allow the needle to slide into the esophagus and the opening of the stomach (b). Stop immediately if one feels blockage from the front end of the needle and re-try. In some cases, one will need to release the mouse and start again 